\title{
Search for Missing Aircraft
}

\author{
Hui $\mathrm{Xu}^{1, \mathrm{a}}$, Yiming $\mathrm{Li}^{2, \mathrm{~b}}$ \\ ${ }^{1}$ Faculty of Mechanical Engineering \& Mechanics, Ningbo University, Ningbo, 315211, China \\ ${ }^{2}$ Faculty of Sciences, Ningbo University, Ningbo, 315211, China \\ email: 459543524@qq.com, bemail:271156266@qq.com
}

Keywords: Multi-objective Optimization; Stochastic Process; Aerodynamics Model

\begin{abstract}
In this paper, a multi-objective optimization model is established for the search of missing aircraft considering the time, efficiency and cost. First, a probabilistic model is developed to determine the distribution of the aircraft's falling zone. Secondly, we provide a fair estimation of cost based on both a drift model and a resource allocation model. Given the data availability, the aforementioned approach has been shown to have the strong capability of optimizing a search scheme and significantly increase the rescue success rate. In addition, the usage of the model including but not limited to those by aircraft and ships and those for the suspected wreckage only by aircraft.
\end{abstract}

\section{Introduction}

The missing Malaysia Airlines Flight MH370 has been one of the most bizarre disappearances in aviation history. After the crash, Malaysia, China, the United States, and other countries immediately launched a large-scale rescue effort. The incident has happened for nearly a year. Though the search zone has been expanded time and again, any wreckage related to the missing flight has not been found yet. Recently, the Prime Minister of Malaysia announced that Flight MH370 ended in the southern Indian Ocean.

At present, the search by aircraft is one of the most common and effective ways for search and rescue (SAR). Different electronic devices or sensors are usually applied in SAR aircraft. It is commonly believed that the combination of these varied electronic devices or sensors has played a significant role in SAR. However, a complete search model does not exist. In this paper, we establish a common model to develop a search program available for the lost plane.

Since the SAR of a missing aircraft is a problem of multi-objective optimization, there are some models which can be used to solve such problems. One of them is the target probability distribution model. This model utilizes the probability model to identify the area where the search target may appear and its probability distribution and further guide the algorithm to choose the optimal search route. The other one is the multi-objective optimization model based on preference. In the multi-objective optimization, as the target dimension is not assimilated, the preference information should be examined by the analysis of control variables so as to make the search more reasonable.

\section{Aircraft Falling Region Simulation}

Aircraft falling region is an approximately rectangular and unevenly distributed irregular graphics and which will affect the searching process. As a result of it, the aircraft falling region should be considered at first.

According to the differential equations, the level of gliding distance of the aircraft $L_{0}$ is 15878 meters. We believe that the course may change when the aircraft falling, but the general trend is to fly forward, and the main scope for a change in the angle of $\left[-90^{\circ}, 90^{\circ}\right]$. We assume that the angle of the aircraft deviated from the original route obedience normal distribution $N(0, \pi / 6)$, the actual level of the whereabouts of the distance is $L=L_{0}+d, L_{0}$ is theoretically gliding distance, $d$ is a deviation. $d$ assumed to obey normal distribution $N(0,4)$. The simulation of it is shown in Fig.1: 


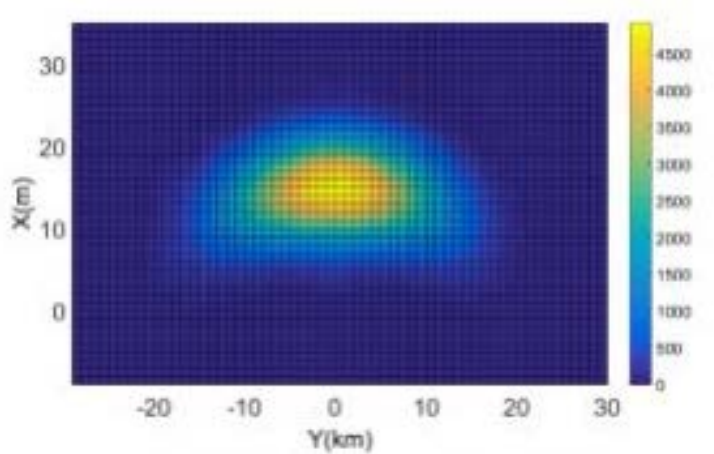

Figure 1(a) the possibility of the drop point

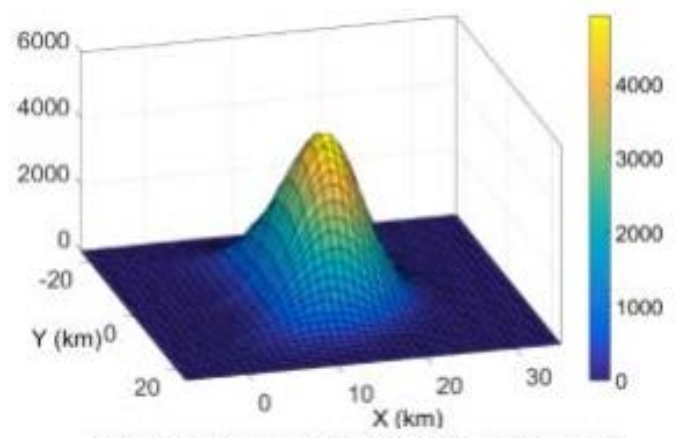

Figure 1(b) the possibility of the drop point

\section{Drift Model}

To identify the best search zone, our usual concern is the possible minimum zone where the search target may appear and the probability distribution within the zone, which is related to not only the position where the aircraft falls but also the impact of the oceanic drift. Because of the rotation of the earth, the object having a relative motion with the earth will be affected by the geostrophic deflecting force, which is also one of the factors that affect the drift. In the long-term search, its cumulative effects need to be taken into account.

As the search time goes by and the oceanic environmental condition changes, the oceanic drift will play an important role in identifying the zone and the probability distribution of the target, which is the basis of the reasonable search direction and the optimized search route.

In a wide sea area, the ocean current usually has fixed general direction and velocity. However, the factors caused by wind direction, wave, and vibration and drift of objects are random and change over time. The random factor of drift is a random number with the uniform distribution between $[0,1]$ and identifies the probability distribution of a random variable in a probabilistic structural analysis. Each random number corresponds to a point-in-time, reflecting that the influence of random factors on the drift is also random.

It is believed that the direction of the random velocity of drift $V_{\text {random }}$ magnitude of the random factor of drift $A$ is also constant. Because $V_{\text {random }}$ is not the velocity of the drifting object itself but is constituted under the influence of external factors, sudden changes of velocity can occur. According to the relation between the velocity and time, in per unit of time, it can be generalized as:

$$
X_{\text {object }}=X_{\text {steady }}+X_{\text {random }}
$$

Where $X_{\text {steady }}$ is the constant displacement of drift, $X_{\text {object }}$ is the displacement of a drifting object, $X_{\text {random }}$ is the random velocity of drift.

With the point where an aircraft falls as the initial point, a rectangular coordinate. The Wikipedia shows that the velocity of ocean currents is generally $0.9-2.8(\mathrm{~km} / \mathrm{h})$. Without losing the generality, in the image of the drift zone, the drift velocity is stable at $2(\mathrm{~km} / \mathrm{h})$ with the direction of $45^{\circ}$, the random velocity of the drift is $1(\mathrm{~km} / \mathrm{h})$ and constant velocity $=1.41 \mathrm{~km} / \mathrm{h}$. The image of the drift zone is shown in Fig. 2:

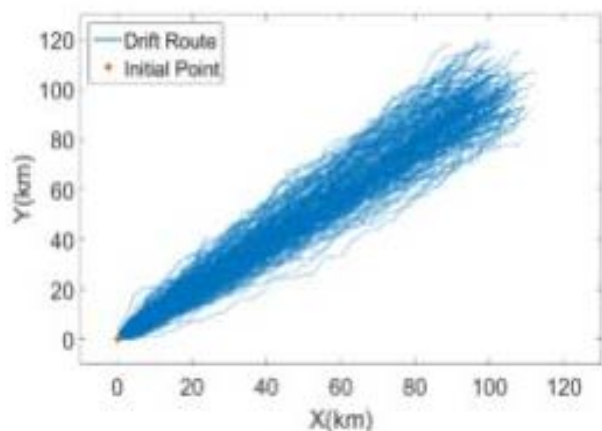

Figure 2(a)

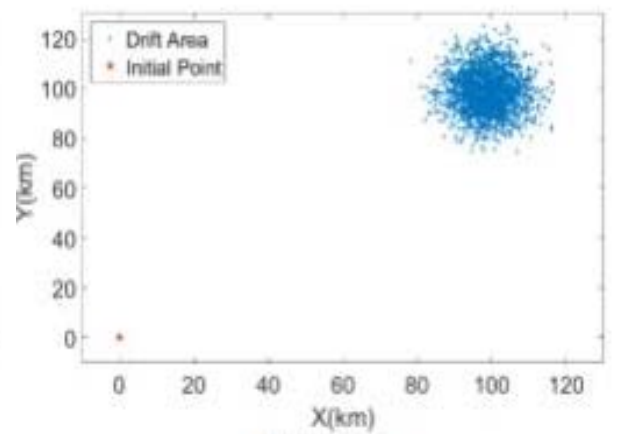

Figure 2(b) 
Referring to the oceanic drift model, it will be introduced to solve the simulation of real problems (Optimal Rectangular Search Distribution Model).

\section{Optimal Rectangular Search Distribution Model}

Since the searching resources that the actual search unit (search aircraft) provides are not infinitely divided, it will take time for each searching partition to allocate searching resources. Besides, the target will make drift motion during the search process[1]. Although the model is complexly resulting from various considerations, the upper range value of the probability of success of all search plans is identified by taking single factors into account. Based on that, the model is reasonably simplified. Specifically, planners of the search are assigned to a search rectangle in the search zone to each search unit in order to maximize its optimality and make the probability of success as close as possible to the theoretical optimal value when its actual operational constraints are met.

\subsection{Function of the Search Rectangle}

The search rectangle which meets the constraints can identify the search route for the search unit in the search rectangle. And the route can be used to calculate the POS for the search[1]. Probability of Success (POS) refers to the probability of finding the target, which depends on two probabilities: 1) the probability of containing (POC), refers to the probability that the search zone contains the search target; 2) the probability of detection (POD), refers to the probability that the search unit detects its target in the search zone. Here it is formulated that: POS=POC $x$ POD.

\subsection{Constraints}

It is assumed that the random particle $Q$ is used to describe the probability distribution of the target's location. The coordinates of the random position particle $q$ are $\left(X_{q}, Y_{q}, w_{q}\right)$ and $\mathrm{q}=1$, $2, \ldots, Q$ in which $\left(X_{q}, Y_{q}\right)$ is the coordinates of the random particle, and $w_{q}$ is the probability of the particle at that location. When the area of $R^{k}$ is $A_{k}$, the set $\Gamma=\left\{q:\left(X_{q}, Y_{q}\right) \in R^{k}\right\}$ represents a set of all the random particles in $R^{k}$, and the width of the $k^{\text {th }}$ e unit's scan is $W_{k}$. Therefore, during the search process, the probability that the search zone contains the search target and the probability that the search target is found are respectively the formula (2) and formula(3) of constraints in the model.

Meanwhile, search rectangles should be not overlapped during the search process. That is to say, the search rectangles which are assigned to search aircraft should not be overlapped. At the same time, given the limitation of available searching resources, the effective search range of the search unit is used for the measurement in the model. In the distribution set of $F$, the distribution function of $f$ is a non-negative function defined in the search space with $f \in F$. However, the POS depends on the choice of distribution functions of $f^{*}$. It is assumed that there is an optimal distribution function of $f^{*}$ which makes the POS reaches its maximum. And so the optimization model can be established as follows[2][3]:

Looking for a $f^{*} \in F$, to make

$$
\max \{\operatorname{POS}[f]: f \in F\}=\operatorname{POS}\left[f^{\prime}\right]
$$

$$
\begin{gathered}
P O S\left[f^{*}\right]=\sum_{k=1}^{n} P_{C}{ }^{k} P_{D}{ }^{k} \\
P_{D}{ }^{k}=1-\exp \left(-\frac{L_{k} W_{k}}{A_{k}}\right) \\
P\left(R^{1} \cap R^{2} \cap \cdots \cap R^{n}\right)=\emptyset, f\left(R^{k}\right)=L_{k}
\end{gathered}
$$

\section{Simulation}

\subsection{Determine the Search Area and Route}

Due to the maritime search and comprehensive consideration of weather and environment conditions (visibility, wind speed, wave height).the Probability distribution of possible locations 
within the target of the search area, search power itself (helicopters, fixed-wing aircraft) as well as with the case, select the appropriate search methods to achieve the best search results. Exercise problems require different types of search aircraft methods, therefore, the search method is carried out at sea. Because the search area is relatively flat, and the aircraft crashed in a vast sea, the specific location uncertain, so the use of parallel lines glanced search mode.

Taking into account the aircraft between the satellite and the communication is not always, at the last received signal as normal airplane flying aircraft, over a period of time is determined prior to the crash, the aircraft may crash at any time, The probable flight distance of the aircraft is $L_{0}$, where $L_{0} \in(0, L)$. Without loss of generality, we consider the plane flying at a speed of $800 \mathrm{~km}$ per hour, flying an hour. The point where the aircraft may fall as shown at Fig .3:

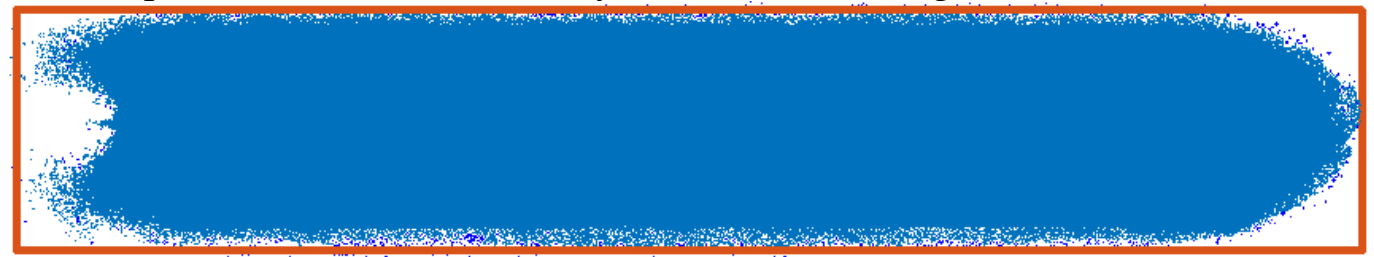

Figure 3 Search zone

Taking into account the plane fell into the water still drift, we appropriate to broaden your search, consider reasonable search area is a rectangle. Aircraft only search within this rectangular area. As long as the drift from entering the detection range of airborne instruments, they think the search is successful.

Compared to the entire rectangular area, tip effects can be ignored. Considered distributed along the $\mathrm{x}$ direction is uniform. In the y direction, Our air crashing placement distribution normality fit in the $y$ direction found a normal distribution $N(30,7.52)$.

Expand the scope of the search that area may exist within the [1000,60] a rectangular area, fall placement probability distribution which is shown in Fig. 4:

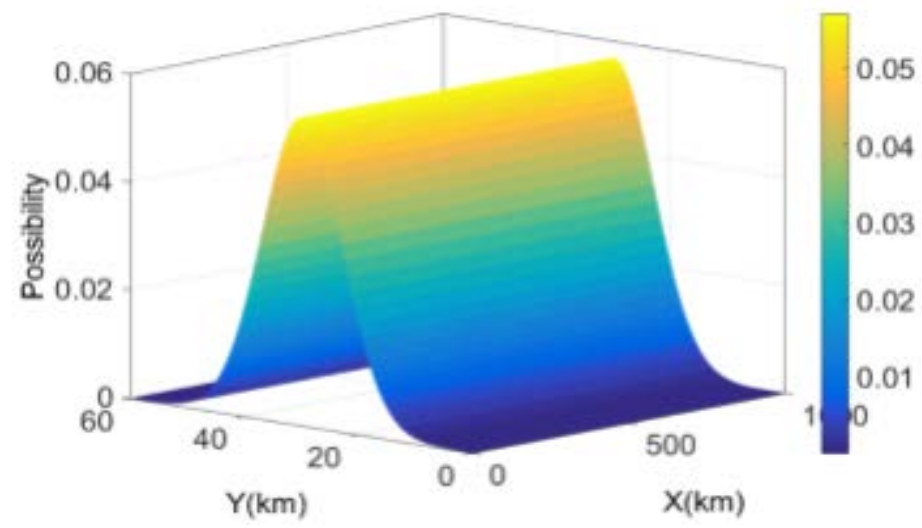

Figure 4 The probability distribution on the search area

\subsection{The Impact of the Success Rate}

Our air crash placement distribution normality fit in the y direction, Simply analysis, in the case of the aircraft can fly always, when various factors changes, the influence of the successful rate of search aircraft, search time, the search efficiency of. There is a constant angle between the direction of the drift velocity and direction of the search area when an airplane falls toward the sea, the drift of sea is also an important factor, without lose of generality, in this simulation considers the angle is $45^{\circ}$. The speed is $0.707(\mathrm{~km} / \mathrm{h})$, the drift velocity randomly selected $0.5(\mathrm{~km} / \mathrm{h})$.

\section{The impact of the crashed aircraft}

$$
\text { Efficiency }=\frac{\text { the rate of success }}{\text { time }}
$$

The impact of the wrecked plane model on the course of rescue operation mainly lies in the searching scope. Search region mainly by the crashed aircraft altitude, horizontal distance fall, fall direction of the decision. When more extensive search area, the use of the longer, the success rate is 
lower, The wreckage of a plane crash when the situation is one of the important factors affecting the success rate.

\section{Search aircraft velocity impact}

The impact of search aircraft mainly reflected on the speed of the aircraft, different flight speed to the rate of success has a significant impact. The speed of the aircraft, simulation results are shown in Fig .5:
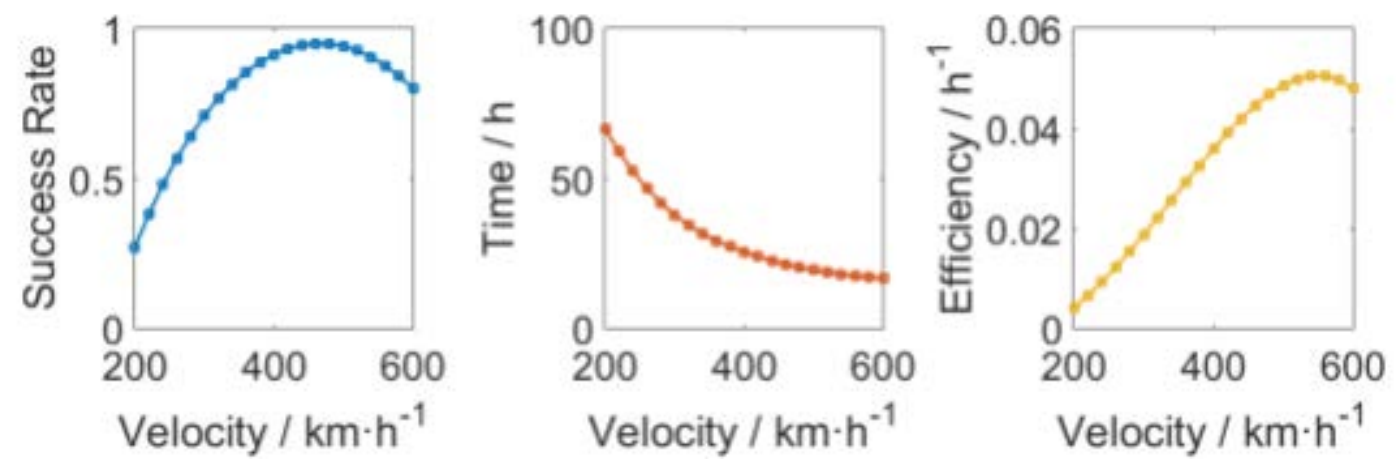

Figure 5 The impact of the velocity

We can see that with increasing search speed, the success rate will be increased, then decreased, but through the entire region continues to decrease with time, search no further increase in efficiency is reached to a certain extent. Choose a reasonable search aircraft, a reasonable choice of flight speed to improve the success rate and reduce the search time of great significance.

Search instrument impact

Affect search instrument mainly reflected in the width of the aircraft search. Simulate aircraft search with the different width. The results are shown in Fig .6:
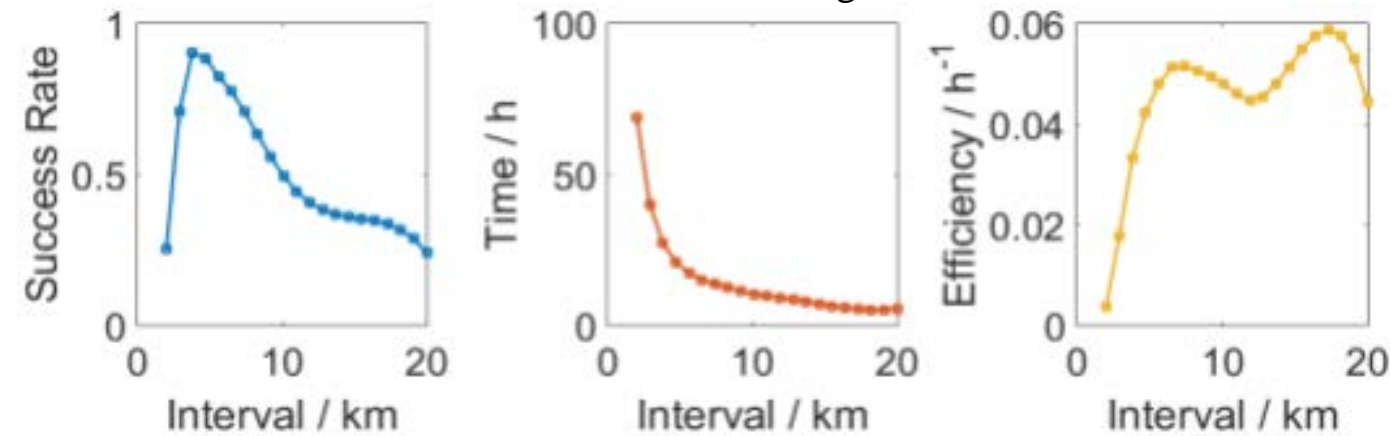

Figure 6 The impact of the width between the trajectory

A more powerful of the search instrument, a more extensive search width, the greater of the success rate, the shorter of the time used, the higher of the efficiency. This guides the development of high-end hardware, which will help the future of search.

\section{The influences of drift velocity}

Random rate and constant rate of drifting have influences on the searching results.

Here come the results by simulating these two rates which are shown in Fig .7 and Fig .8. When a constant rate of drifting is fast, the searching goal leaves the area to be searched before the searching planes search all over around, which greatly decreases the success rate, increases the time cost and decreases the efficiency. 

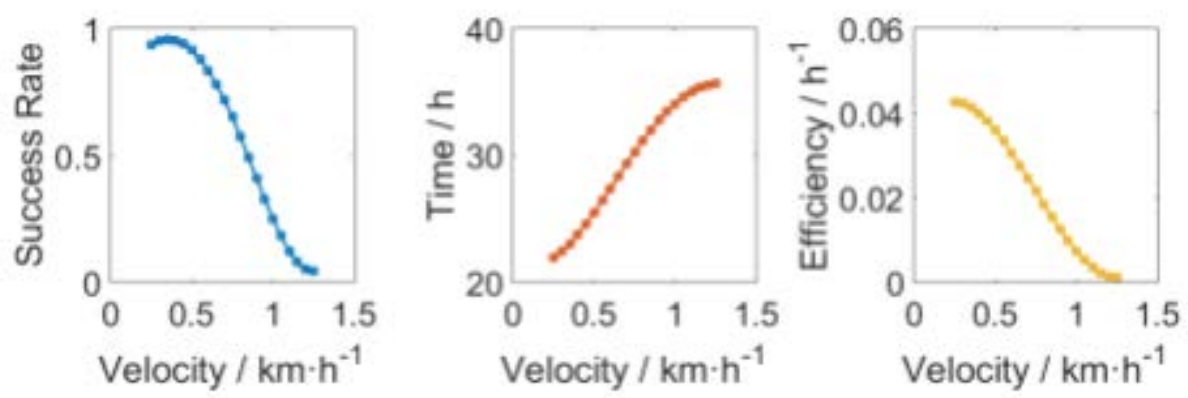

Figure 7 The impact of the constant drift velocity
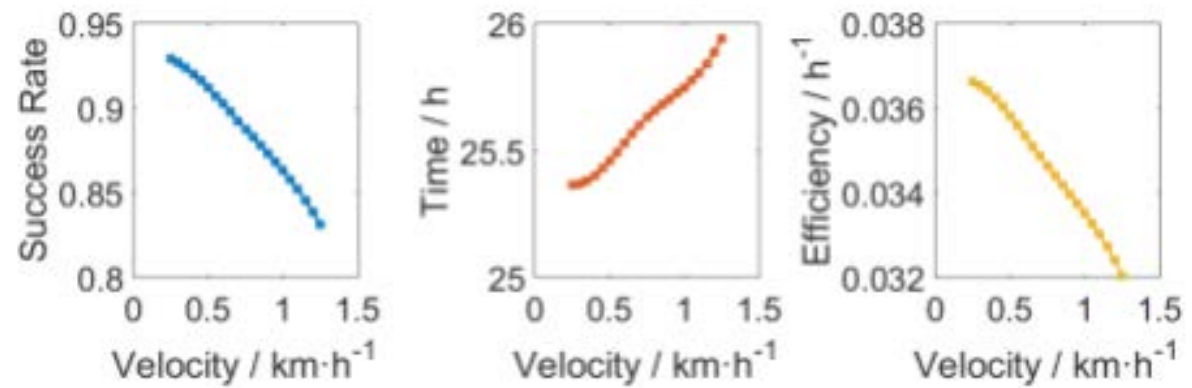

Figure 8 The impact of the random drift velocity

When the random rate of drifting is fast, the success rate falls down only a little, and the time cost has an invisible change.

\section{Conclusion}

A model based on multi-objective optimization preferences is established to determine the drop probability distribution in the search range. Then, changing the basic parameters: the search width, the search speed, search path distance, environmental factors to simulate rescue success rate and search efficiency under different conditions. Finally, analyzed to obtain in the case of search and rescue planes to guide the rescue unfold in reality working to develop a reasonable search and rescue program.

\section{Acknowledgement}

We are grateful to Doctor Songjing Wang from the faculty of Sciences of Ningbo University for useful discussion.

\section{References}

[1] S. AcharyaN. RanarahuJ. K. DashM. M. Acharya 《Journal of intelligent \&amp; fuzzy systems: Applications in Engineering and Technology》, 20142

[2] Javaid, S.Ansari, S.I.Anwar, Z. 《Opsearch: Journal of the Operational Research Society of India》, 20132

[3] http://cn.mathworks.com/discovery/multiobjective-optimization.html

[4] http://en.wikipedia.org/wiki/Aerodynamics

[5] http://en.wikipedia.org/wiki/Sea_Drift_(Delius

[6] http://www.dow.com/locations/texas/seadrift/

[7] Breivik OAllen AA 《Journal of marine systems: journal of the European Association of Marine Sciences and Techniques》, 2008 1/2 